Classification

Physics Abstracts

61.16D - 61.70D - 78.70D

\title{
Spatially resolved electron energy-loss near-edge structure analysis of a near $\Sigma=11$ tilt boundary in sapphire
}

\author{
John Bruley \\ Max-Planck-Institut für Metallforschung, Institut für Werkstoffwissenschaft, Seestraße 92, D-7000 \\ Stuttgart 1, Germany
}

(Received 11 December 1992, accepted 22 February 1993)

\begin{abstract}
Spatially resolved electron energy-loss spectra have been recorded from the same $\Sigma=11$ grain boundary of a high purity sapphire $\left(\alpha-\mathrm{Al}_{2} \mathrm{O}_{3}\right)$ bi-crystal that had previously been examined by high resolution electron microscopy. The energy-loss near-edge structure (ELNES) on the aluminium $\mathrm{L}_{2,3}$ edge of the bulk $\alpha-\mathrm{Al}_{2} \mathrm{O}_{3}$ is consistent with previously published $\mathrm{x}$-ray absorption near-edge spectra (XANES). The presence of the grain-boundary introduces additional fine structure on the $\mathrm{L}_{2,3}$ edge, which is extracted from the dominant bulk spectrum using the chemical-standard "difference" method. A comparison with various spectral "fingerprints" suggests that the structural environment of the $\mathrm{Al}$ cations within the boundary plane has a reduced point-group symmetry and coordination number. Quantitative analyses of spectral intensities indicate that the equivalent of a full monolayer of $\mathrm{Al}$ cations is involved in the structural change at the boundary. These experimental observations are consistent with a recently calculated low energy relaxed grain-boundary structure.
\end{abstract}

\section{Introduction.}

Energy-loss near-edge structure (ELNES) $\left({ }^{1}\right)$ is sensitive to the atomic coordination, symmetry, chemical bonding, and ionicity and in favorable circumstances can be recorded with a lateral resolution approaching the optimum instrumental beam size [1]. With a parallel acquisition energy loss spectrometer (PEELS) fitted to a dedicated field emission Scanning Transmission Electron Microscope (STEM), column-by-column Ångstrom-scale characterization of electronic structure and chemistry having near-single atom sensitivity is now a realistic goal [1-4]. The general technique has become known as Spatially Resolved Electron-Energy -Loss Spectroscopy or SREELS.

Ultra-pure and magnesium-doped sapphire $\left(\alpha-\mathrm{Al}_{2} \mathrm{O}_{3}\right)$ bi-crystals having a near coincident site lattice (CSL) $\Sigma=11,35.2^{\circ}[\overline{2} 110]$ tilt boundary with the $(01 \overline{1} 1)$ plane have recently been the focus of a combined experimental and theoretical study designed to probe the atomic structure of grain boundaries in ionic oxides $[5,6]$. By comparing high resolution electron micrographs (HREM) to image simulations of grain boundary structures which had been calculated by atomic simulation, it was concluded that the minimum energy and most densely packed structure provided the "best" and consequently, the most "likely" match. According to this structural model

$\left({ }^{1}\right)$ or XANES, the analogue to ELNES in $\mathrm{x}$-ray absorption spectroscopy. 
the coordination, symmetry and bond-lengths and angles of all of the $\mathrm{Al}$ ions located on the interface plane are to some extent affected. In the model, there are 12 distinct Al sites. So far there have been no attempts to calculate electron band-structure diagrams for this or any other grain boundary structure of sapphire.

The same bi-crystals were considered suitable candidates for a SREELS study. The occurrence of distinctive short range "coordination- or chemical-fingerprints" [7-9] or "shape-resonances" [10] in the near-edge structure of the $\mathrm{Al} \mathrm{L}_{2,3}$ edge in various aluminium oxide materials highlights the feasibility of characterizing the structure of simple interfacial units. This work represents the first such attempt to examine specific alumina grain-boundaries by SREELS and to relate the surface sensitive ELNES to electronic and atomic structure. In this study attention is only given to the ultra-pure sample; an attempt to detect $\mathrm{Mg}$ in the doped material and an investigation of the resulting grain boundary structure will be discussed elsewhere.

Near CSL $\Sigma=11$ boundaries have been previously identified in sintered [11] and commercial aluminas [12], although in those cases the boundary plane was not faceted on $\{10 \overline{1} 1\}$ but on $\{10 \overline{1} 0\}$ and $\{10 \overline{1} 2\}$ planes.

\section{Experimental meethods.}

2.1 THE SAMPLE. - The near- $\Sigma 11$ bi-crystals were supplied by Dr. P.A. Morris, of the Massachusetts Institute of Technology, USA. They were fabricated as part of a project to develop procedures for producing high-purity single, bi- and poly-crystals of $\alpha-\mathrm{Al}_{2} \mathrm{O}_{3}$ [13]. The bi-crystal was melt-grown using two seed crystals which had been purified by multiple zone refinement, using a $\mathrm{CO}_{2}$-laser float zone technique. The product was reported to contain less than $38 \mathrm{ppm}$ cation impurity and less than $59 \mathrm{ppm}$ total impurity. The same cross-sectioned TEM sample previously prepared and examined in the HREM was used for this study [6].

2.2 INSTRUMENTATION. - Spatially resolved electron energy-loss spectroscopy (SREELS) was carried out using a VG Microscopes' model HB501 STEM fitted with a cold field emission source and a Gatan model 666 PEELS. The microscope was operated at $100 \mathrm{keV}$, with the first condensor lens switched off and the second condensor lens focussed at the selected area aperture plane. The angular acceptance aperture of the spectrometer was $8 \mathrm{mrad}$ and the beam convergence angle was about $8 \mathrm{mrad}$. Under such illumination conditions a beam current of about $0.2 \mathrm{nA}$ through a probe diameter of about $0.5 \mathrm{~nm}$ is expected, although this was not explicitly measured here.

2.3 DATA ACQUiSiTION. - The specimen was tilted such that the beam was incident along the [2110] zone axis and parallel to the near CSL $\Sigma=11$ grain boundary plane. In this orientation the projection of the boundary extends along [0111]. Regions of foil less than $30 \mathrm{~nm}$ thick were selected.

Specimen movement during analysis is an obvious cause for concern. On occasions the interface was stable to drift for durations exceeding a minute, whilst on others there was a linear drift rate of several nanometers per minute. The cause for this unpredictable movement is probably due to slight specimen charging effects. As a precautionary measure for the analysis, the boundary was viewed before and after each spectrum acquisition to ensure both specimen and beam stability. During stable periods the beam could be placed onto the interface with an accuracy of better than $0.3 \mathrm{~nm}$ for the time required to record the EELS data. Most spectra were recorded with a stationary beam placed at various locations on and near to the interface. Some spectra were also acquired with the beam rastering over a 1 by $1.3 \mathrm{~nm}^{2}$ area centered on the interface. Scanning the 
beam minimizes specimen damage and enables visual inspection of the boundary during analysis, enabling active specimen drift correction using electrical beam deflection coils.

Radiation damage affects the atomic structure of the boundary and can significantly alter the near-edge structure, which is a severe limitation in the study of $\alpha-\mathrm{Al}_{2} \mathrm{O}_{3} \cdot \alpha-\mathrm{Al}_{2} \mathrm{O}_{3}$ is known to be sensitive to damage, see for example reference [14] and references therein. In the current study, damage was monitored by recording a time sequence of spectra from the same region of sample. Typically, no significant variations are observed between spectra recorded in less than about 30 seconds under the illumination conditions quoted. By scanning the beam parallel to the interface during analysis, this time could be extended.

The acquision time between each diode readout (typically $5 \mathrm{~s}$ ) was adjusted such that about $10^{4}$ photodiode counts per channel (corresponding to $2 \times 10^{5}$ incident electrons) were recorded with a $0.1 \mathrm{eV} / \mathrm{channel}$ energy dispersion. A fixed diode read-out pattern was removed from each spectrum. No attempt was made to correct for the channel-to-channel gain variation, which is about $1 \%$ for the Gatan detector [15]. Each spectrum is constructed from the sum of up to 6 photodiode readouts, representing a 30 second total acquisition time. The energy resolution of the zero-loss peak was between 0.4 and $0.6 \mathrm{eV}$ f.w.h.m. for these experimental conditions. The resolution is expected to be similar at the $\mathrm{Al}-\mathrm{L}_{2,3}$ edge.

2.4 DATA ANALYSIS: THE STANDARD "DIFFERENCE" MODE. - For a weak signal-to-background ratio, the optimum detection sensitivity is limited by the statistical variance (or uncertainty) in the background [16]. The standard "difference" method provides a convenient way to accurately model and extract a non-smooth energy dependent background. The background is approximated by a reference spectrum taken from the nearby bulk matrix. At interfaces and defects the technique is equivalent to performing a "first-derivative" with respect to a real-space displacementtypically perpendicular to the interface-and so it is analogous to the more common first and second energy difference modes where differences in intensity are measured with respect to a small shifts along the energy-loss scale [17]. The standard (or real-space) "difference" provides similar advantages over the normal spectrum mode to those reported for the "energy-difference" modes, namely a significant improvement to the signal-to-background ratio, and the non-propagation of detector artifacts. An additional benefit of the real-space "difference" technique is the retention of the ELNES, enabling studies of chemical bonding along with quantitative analysis using the standard ionization cross-sections. Recently, the method was used to detect and quantify the near-edge structure of $1 / 10$-monolayer of nitrogen segregated to planar defects in diamond [18]. For the study of radiation sensitive materials, as is the case here, the real-space "difference" mode requires that the sampled region only be exposed to a minimum dose, the second spectrum being recorded at a separate location. This is not true for the energy "difference" mode where both spectra need to be recorded at one location.

A series of $\mathrm{Al} \mathrm{L}_{2,3}$ edges were recorded in the energy range from 50 to $150 \mathrm{eV}$ with an energy dispersion of $0.1 \mathrm{eV} / \mathrm{ch}$ annel. The edge-thresholds were aligned in energy and smooth backgrounds of the form $E^{-r}$ (fitted in a $20 \mathrm{eV}$ window before the edge) were subtracted from the data. The real-space "difference" of the grain-boundary was formed by subtracting from the boundary spectrum a normalized bulk $\alpha-\mathrm{Al}_{2} \mathrm{O}_{3}$ spectrum, recorded in the neighboring region of crystal. The correct choice of the normalization factor for the bulk spectrum is rather subjective and depends upon the system chosen for investigation. In the grain boundary system presented in this work, the scattering leading to the $\mathrm{Al}-\mathrm{L}_{2,3}$ ELNES in $\mathrm{Al}_{2} \mathrm{O}_{3}$ is dominated by short range (nearest-neighbor) bonding effects [7-10]. Thus it should be possible to decompose the measured ELNES into several linearly independent components arising from the subset of atomic arrangements exposed by the electron beam. According to this assertion, a suitable definition for the normalization factor for the background spectrum is given by the condition that the bulk spectrum equals the contribution 
of all $\mathrm{Al}$ ions possessing bulk-like chemical bonding in the grain-boundary spectrum.

The real-space "difference" method is schematically illustrated in figure 1 . According to the simplest bimodal distribution model, the spectrum intensity, $l_{\mathrm{t}}$, recorded with the beam located on the boundary is given by the sum of two partial intensities; $l_{\mathrm{b}}$ and $l_{\mathbf{x}}$, where $l_{\mathrm{b}}$ and $l_{\mathrm{x}}$ are proportional to the number of aluminium ions located on bulk sapphire sites and on grain-boundary sites respectively. With the electron beam displaced onto the perfect crystalline region, the number of irradiated aluminum ions gives rise to an intensity $l_{\mathrm{a}}$. A suitable definition for the normalization factor $(f)$ is thus given by the condition that $f$ times $l_{\mathrm{a}}$ is made equal to $l_{\mathrm{b}}$. The real space "difference" is then formally given by the expression, $l_{\mathrm{x}}=l_{\mathrm{t}}-f . l_{\mathrm{a}}$.

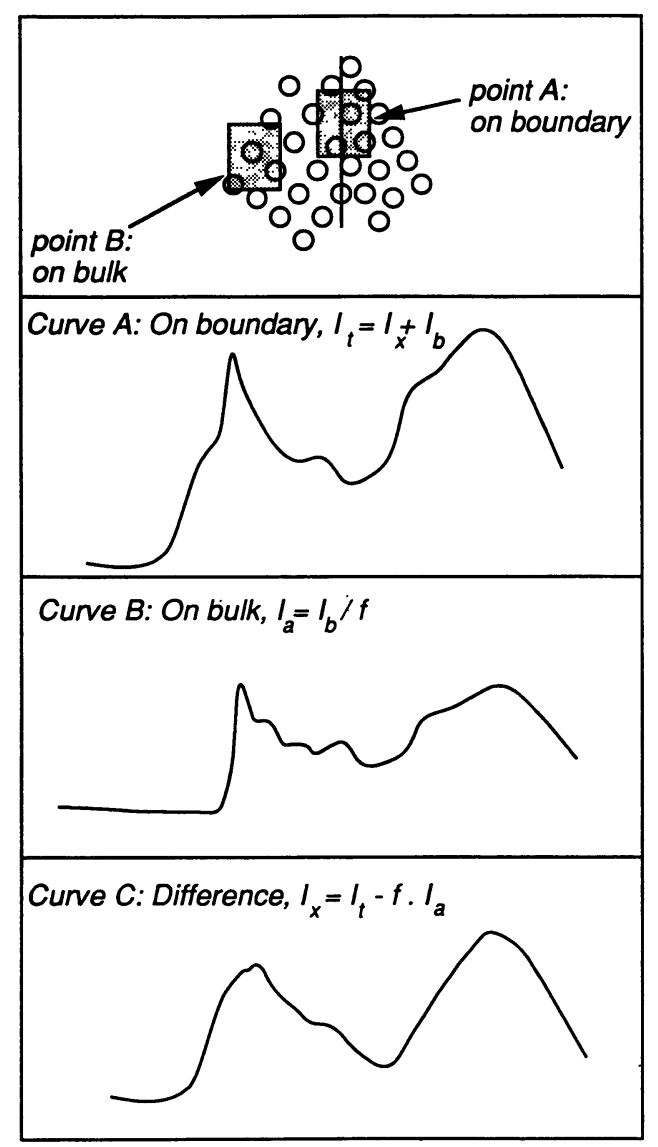

Fig. 1. - A schematic to illustrate the definition of a real-space "difference" spectra. The spectrum recorded at the boundary (curve $\mathrm{A}$ ) is composed of two parts, one from atoms situated on the boundary and the second from atoms within the bulk. The standard spectrum (curve B) recorded beside the boundary is normalized to match intensity of the second component. The difference (curve $\mathrm{C}$ ) reveals the boundary component.

In this work the normalization factor $f$ for the "difference" is determined by trial-and-error, by matching the step-height at the edge threshold located at $78 \mathrm{eV}$ and assessing the smoothness of the "difference" at 79 and $100 \mathrm{eV}$. Sets of features in energy regions characteristic of the sapphire 
ELNES signify either incorrect energy alignment (which is equivalent to forming a "first difference" spectrum) or unsuitable normalizing factor. In almost all cases, normalization could be achieved with an accuracy of better than $\pm 10 \%$ and alignment to \pm 1 channel. An improvement to this approach would require cross correlation of the spectra and multiple least squares fitting.

\section{Results.}

Figure 2 displays two $\mathrm{Al} \mathrm{L} \mathrm{L}_{2,3}$ edge spectra: The first was recorded with the beam on the $\alpha-\mathrm{Al}_{2} \mathrm{O}_{3}$ matrix (curve a) and the second with the beam centered on the $\Sigma 11$ grain boundary (curve b). The bulk $\alpha-\mathrm{Al}_{2} \mathrm{O}_{3}$ spectrum is consistent with previously recorded EELS data of sapphire [7, 19], and higher energy-resolution $x$-ray absorption data [20-22].

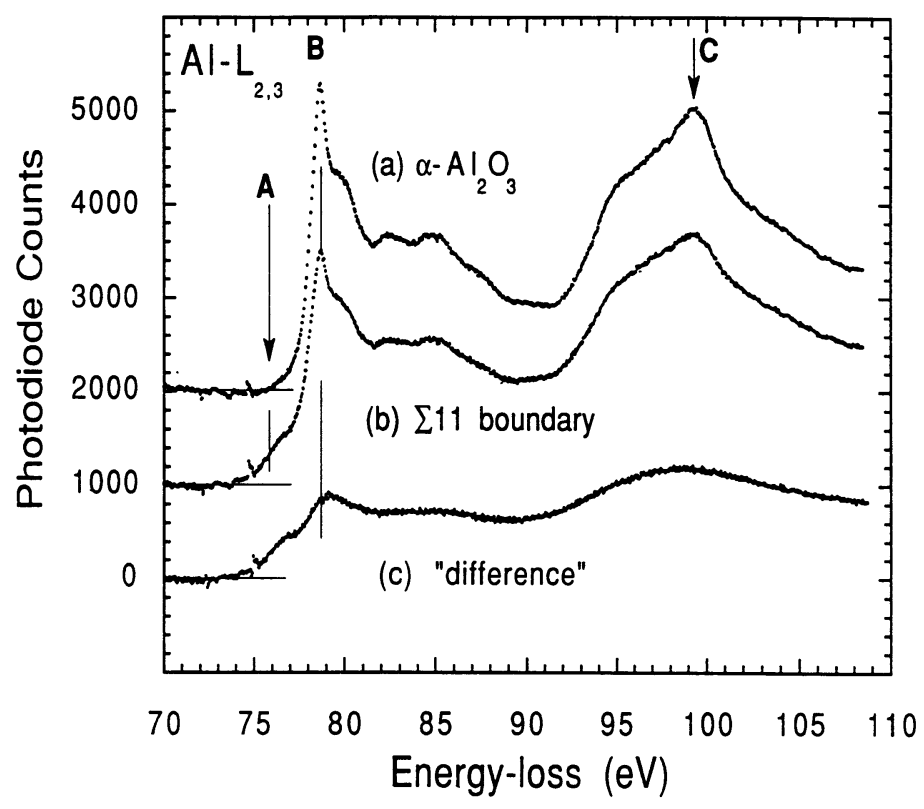

Fig. 2. - The Al- $\mathrm{L}_{2,3}$ for bulk $\alpha-\mathrm{Al}_{2} \mathrm{O}_{3}$ (a), $\Sigma 11$ grain-boundary (b) and the standard "difference" (c). The difference is due to the altered bonding of $\mathrm{Al}$ atoms at the boundary. The assignment of the marked features $\mathrm{A}, \mathrm{B}$, and $\mathrm{C}$ is given in the text.

The similarity between curves (a) and (b) indicate that the modified atomic structure at the boundary only weakly influences the electronic structure or bonding. Two subtle differences are evident upon inspection: The first is the appearance of a weak shoulder in curve b (marked A) preceding the dominant $78.8 \mathrm{eV}$ excitonic absorption. The second is the increased intensity ratio between the peak at $99.5 \mathrm{eV}$ (peak-C) and that at $78.8 \mathrm{eV}$ (peak-B). Normalization and subtraction 
of the matrix spectrum from the interface spectrum yields the standard "difference" (curve-c), making apparent a grain-boundary sensitive near-edge structure. The threshold energy (position at half-height) of the shoulder (A) appearing at the leading edge of this grain-boundary spectrum is $75.8 \mathrm{eV}$ and there is an inflection point at $77 \mathrm{eV}$. The energy of the peak $\mathrm{B}$ is shifted up $79.1 \mathrm{eV}$ and that of the much broader absorption at $\mathrm{C}$ is shifted down to $98.6 \mathrm{eV}$. The shape is reproduced at other locations along the boundary as demonstrated in figure 3. There is some variability observed in the exact energy positions $( \pm 0.4 \mathrm{eV})$, indicating non-uniformity in the electronic structure along the boundary. A systematic study would need to be carried out to ascertain if such variability occurs with a periodicity along the grain-boundary.

Albi2- $\sum 11$ grain boundary

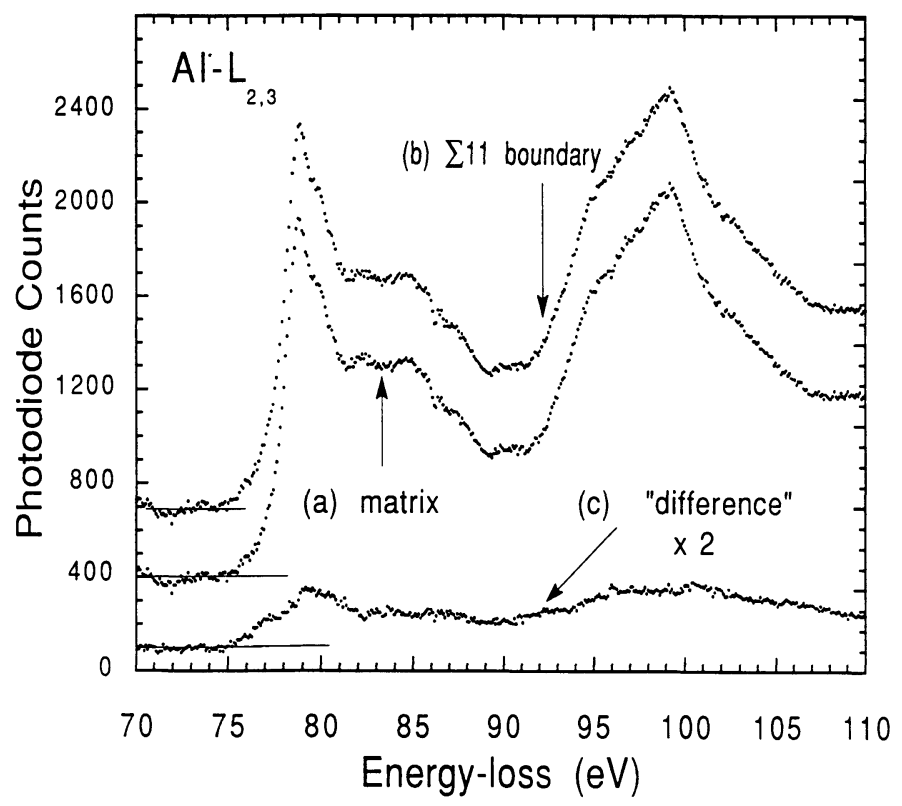

Fig. 3. - Spectra recorded at a second point along the boundary, highlighting reproducibility of the technique. Similar "difference" spectra were recorded at five separate positions.

An alternative definition for the normalization factor $f$ would be to normalize the total integrated intensity of the bulk spectrum to that of the boundary spectrum, such that $f \times l_{\mathrm{b}}=l_{\mathrm{t}}$. The integrated intensity of the "difference" over the near edge region normalized in this way is thus equal to zero. As long as the composition and bonding of the $\mathrm{Al}$ ions do not change too significantly, it is a reasonable assumption that the oscillator strengths obey a partial sum rule independent of local bonding. This being the case, the integrated intensities of $l_{\mathrm{t}}$ and $f \times l_{\mathrm{b}}$ are proportional to the same total number of $\mathrm{Al}$ ions. The structure of this alternatively defined "difference" spectrum is useful in that it reveals the influence of the boundary on the ELNES, highlighting chemical shifts and changes to the unoccupied density of states. A "difference" spec- 
trum generated according to this normalization criterion is presented a curve-b in figure 4 . For comparison, curve-a represents the standard "difference" spectrum obtained using the normalization criterion defined previously. Curve-b illustrates that the ELNES redistribution produces increased intensity forming a low energy peak centered on $76 \mathrm{eV}$ (region-1). This is possibly connected to a downward shift of the vacant $\mathrm{Al}$ p-states from the $78 \mathrm{eV}$ domain which has lost intensity. The broader bands between 87 and $93 \mathrm{eV}$ (region-2) and above $100 \mathrm{eV}$ (region-3) possibly reflect rehybridization and greater dispersion of vacant higher lying poorly localized $\mathrm{Al} \mathrm{d}$ orbitals. The interpretation of these $\mathrm{Al}-\mathrm{L}_{2,3}$ ELNES features is discussed in section 3.

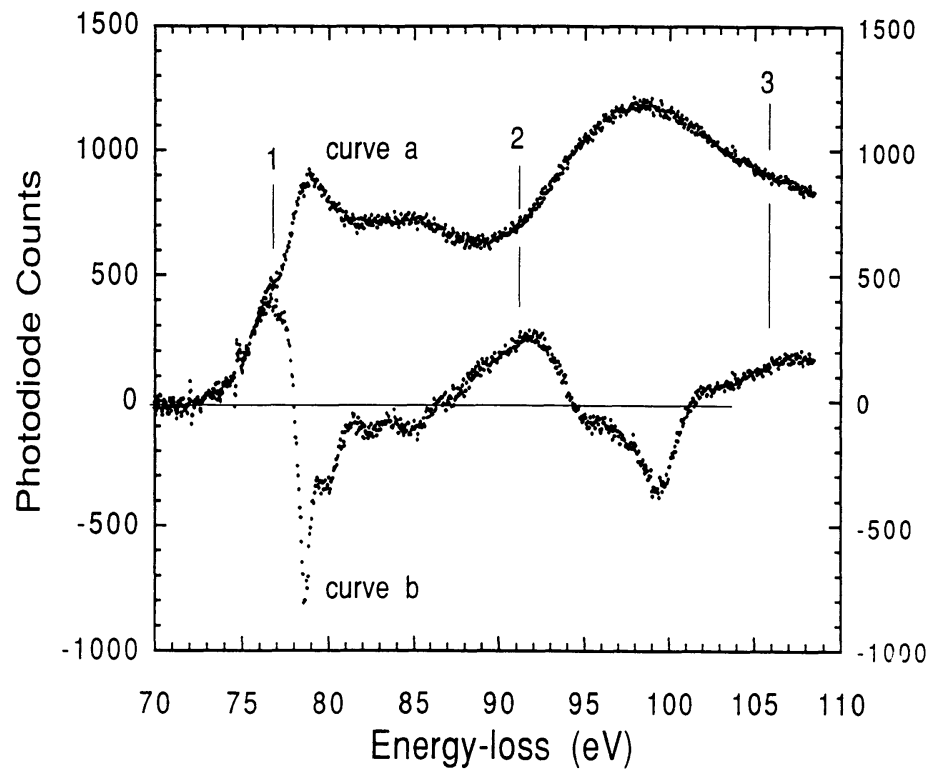

Fig. 4. - Using the normalization criterion that the net intensity of the "difference" spectrum equals zero, the near-edge-structure exposes those energy regions where a redistribution of oscillator strength exists.

The integrated intensity of the "difference" component within a $20 \mathrm{eV}$ window at the edge compared to that of the $\alpha-\mathrm{Al}_{2} \mathrm{O}_{3}$ background intensity is a measure of the relative number of $\mathrm{Al}$ atoms whose bonding is affected by the presence of the boundary. The measured ratios at different locations of the beam are given in table I and range between 0.1 and 0.8 , having an average value 0.38 with a standard deviation of 0.25 .

In figure 5 the difference spectrum is compared to the standard spectrum of amorphous alumina and published XANES calculations. The calculated curves represent tetrahedrally and octahedrally coordinated complexes and are taken from results of single-shell multiple scattering XANES calculations for the $\mathrm{Al}$ cation surrounded by 4 and $6 \mathrm{O}$ anions respectively [8]. There is a pronounced resemblance between the standard "difference" ELNES and that of an amorphous alumina film formed by anodic oxidation of aluminium. The amorphous alumina spectrum was recorded using a very short acquisition time $(<1 \mathrm{~s})$ and a reduced current density (high spatial resolution is not necessary) in order to avoid damage. Such amorphous aluminas are very sensitive and usually crystallize upon intense irradiation [23]. 
Table I. - The ratio of "difference" intensity to the bulk intensity, integrated over a $30 \mathrm{eV}$ window at the edge.

$\begin{array}{ll}\text { spectrum \# } & l_{\mathbf{x}} / l_{\mathrm{B}} \\ 1 & 0.1 \\ 2 & 0.2 \\ 3 & 0.5 \\ 4 & 0.8 \\ 5 & 0.3\end{array}$

Average $\quad 0.38 \pm 0.25$

The influence of electron irradiation on the ELNES was investigated. Figure 6 displays data recorded and the beam located on one location for 10 seconds ( $5 \mathrm{~s}$ acquisition), 30 seconds ( $5 \mathrm{~s}$ acquisition), 1 minute (10 s acquisition), 2 minutes (15 s acquisition) and 5 minutes (60 s acquisition). After 1 minute there is a clear indication of radiation damage to the $\alpha-\mathrm{Al}_{2} \mathrm{O}_{3}$, which is marked by the appearance of the characteristic $\mathrm{L}_{2,3}$ edge of metal having an edge threshold energy of $72.0 \mathrm{eV}$. There is little or no sign of damage for times less than this.

Oxygen K-edge spectra were also recorded at and besides the boundary but these data revealed no interface sensitive "difference" component. To record Al-K edge data, radiation times exceeding 2 minutes were necessary to achieve reasonable counting statistics. This is longer than the time required for excessive damage and so the data are not considered further.

\section{Discussion.}

4.1 BULKSAPPHIRE $\mathrm{Al} \mathrm{L}_{2,3}$ EDGE. - The principle aim of this work is to investigate the bonding and structure of the near CSL $\Sigma=11,35.2^{\circ}$, [2110] tilt boundary by application of the chemicalstandard "difference" ELNES technique. Despite considerable attention, there remains ambiguity concerning the interpretation of this near-edge structure of the bulk $\alpha-\mathrm{Al}_{2} \mathrm{O}_{3}$ phase. To date, the many electronic-structure calculations have been unable to accurately model the edge-shape. The difficulty lies in part with the large number of atoms and complexity of the corundum unit cell and in part with the influence of the $2 p$ core-hole excitonic interaction. However, because of the localized nature of the chemical bonding, some insight has been gained by reference to the $\mathrm{X} \alpha-\mathrm{SCF}$ Molecular Orbital (MO) calculations of the octahedrally coordinated $\left(\mathrm{AlO}_{6}\right)^{9-}$ [24]. From this MO-scheme, the lowest energy peak at $79 \mathrm{eV}$ (peak B in Fig. 2) would be assigned to transitions from the Al-2p core-level into unfilled $7 \mathrm{a}_{1 \mathrm{~g}} \mathrm{MOs}$, the $7 \mathrm{a}_{1 \mathrm{~g}}$ being constructed predominantly from Al-3s orbitals. A more recent small cluster CNDO MO calculation, described in reference [22], enabled a better match to the data up to $10 \mathrm{eV}$ above the threshold energy. The one-electron band structure calculations are in general agreement that the lowest unfilled conduction band is composed of $\mathrm{Al} 3 \mathrm{~s}$ and $3 \mathrm{p}$ atomic orbitals with some minor covalent admixture of oxygen s orbitals [25-30].

The intense threshold peak at $78.7 \mathrm{eV}$ for sapphire has been identified with a core-exciton and would thus explain the failure of one-electron band theory to model the edge $[10,22,31,32]$. However, all the major features of the near-edge structure for octahedrally coordinated Al cations have been simulated using a multiple scattering XANES computation which did not include the core-hole excitonic interaction [8]. Thus a calculation providing the correct symmetry and site 


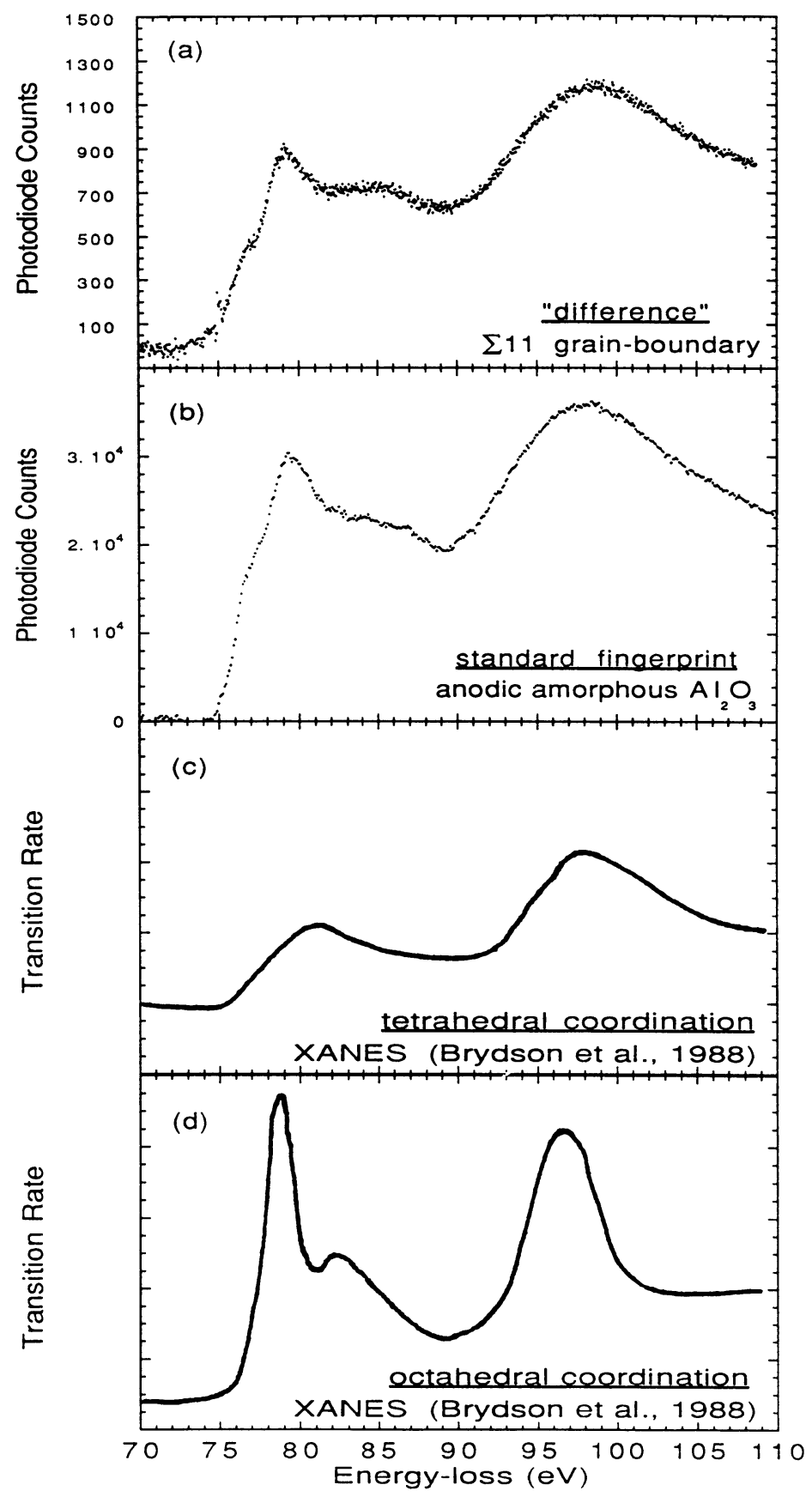

Fig. 5. - The standard difference spectrum at the $\Sigma 11$ grain boundary compared with the spectral "fingerprint" of anodic amorphous alumina and the XANES computed edges for tetrahedral and octahedral clusters of $\mathrm{Al}$ surrounded by 4 and $6 \mathrm{O}$ anions respectively, reproduced from reference [8].

projection of the density of states using one-electron band structure theory may be adequate to explain the observed near-edge structure of bulk and boundary spectra. 


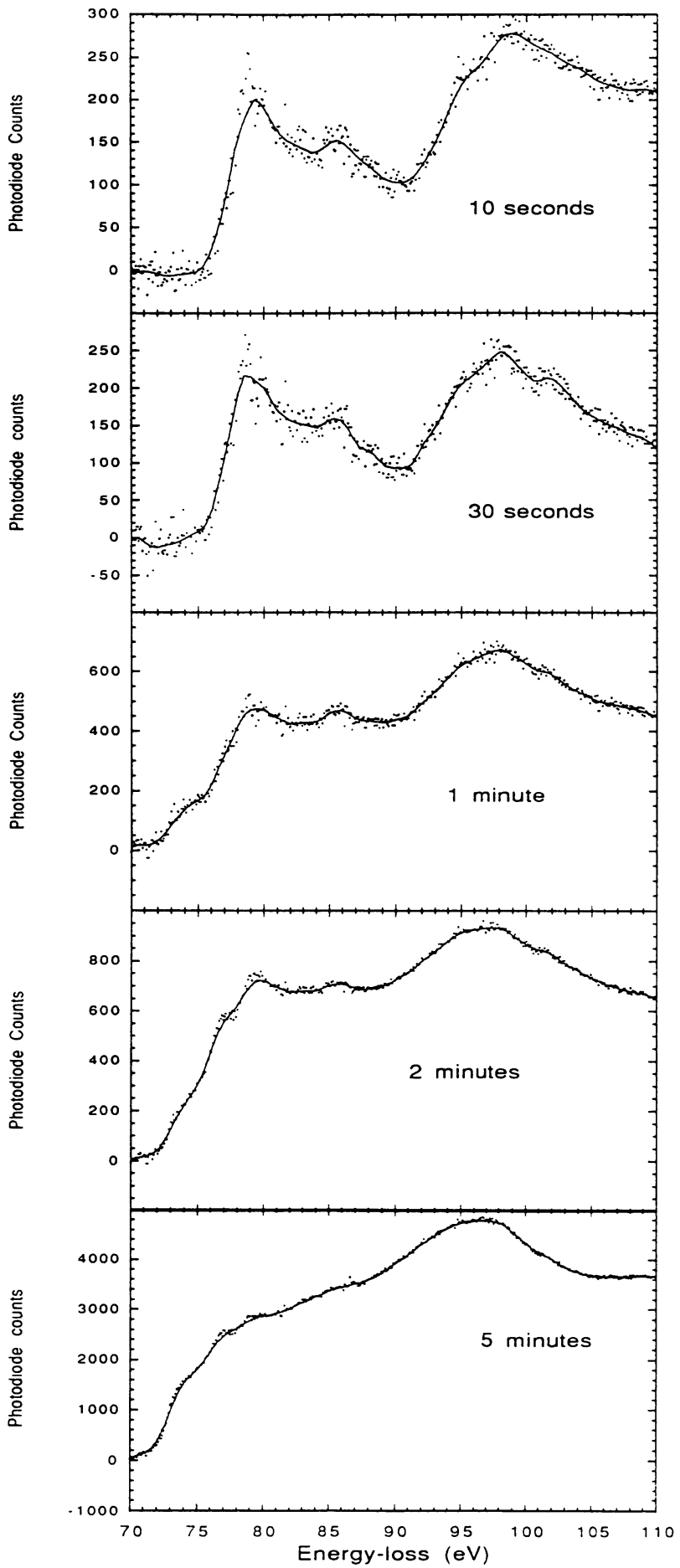

Fig. 6. - A series of spectra illustrating the effect of radiation time on the grain boundary difference spectra. After 1 minute there is evidence of structural modification. The rise of an edge at $72 \mathrm{eV}$ indicates metallization. 
Until the accuracy and predictive capability of detailed electronic calculations for the interpretation of ELNES are improved, the optimum route for interpreting spatially resolved near edge structures is by appeal to a library of "chemical fingerprint" spectra. In combination with other atomic structure probes such as HREM, spatially resolved ELNES provides detail on the local changes in electronic structure and bonding.

4.2 INTERFACE SENSITIVE $\mathrm{Al} \mathrm{L}_{23}$ ELNES COMPONENT. - The real-space “difference" component of the sapphire grain-boundary spectrum reflects changes in the structural environment of $\mathrm{Al}$ ions. Modelling the grain boundary structure in an ionic ceramic such as $\alpha-\mathrm{Al}_{2} \mathrm{O}_{3}$ is complicated by the requirement that the distance between nearby ions of like charge should be maximized and ions across the interface should be of opposite charge. There are various mechanisms through which the boundary can re-configure to lower the total energy. These include structural or atomic rearrangements, composition modifications via impurity or point defect (i.e. vacancy) segregation, and charge transfer.

The lowest energy atomistic structure of the near CSL $\Sigma=11$ boundary calculated by Kenway [5] which best fits HREM images [6] is an attractive explanation for these ELNES observations. The Kenway relaxed structural model of the near $\Sigma=11$ tilt boundary is depicted in figure 7 (viewed in projection along the $[\overline{2} 110]$ direction). The details of the atomic relaxations are too complex to describe in detail in this study, and will be the topic of a forthcoming paper by Kenway. By inspection of the figure, it is possible deduce that the $\mathrm{Al}$ ions situated close to the boundary occupy modified environments with variable coordination in a more open structure. The typical region of boundary sampled by the electron beam in these measurements is indicated by the large black circle.

To explore this and alternative possibilities, either a suitable chemical "fingerprint" from a known structure needs to be identified or a electronic density-of-states calculated. The first option which concerns comparison to a suitable "fingerprint", has not been possible to date, because there are no materials that possess a bulk structure resembling the detailed arrangements of the atoms at the boundary proposed by Kenway. The spectrum from amorphous anodic $\mathrm{Al}_{2} \mathrm{O}_{3}$ does appear to provide an excellent "fingerprint" (see Fig. 5) but its atomic structure is also uncertain. It is feasible that the interatomic forces influencing atomic relaxation within the grain-boundary region are similar to those that control the short range order of an amorphous phase. If true, the grain boundary could be described as a monolayer thick amorphous grain-boundary film. Separate investigations of the structure of the anodic $\mathrm{Al}_{2} \mathrm{O}_{3}$ films indicate that between 20 and $80 \%$ of the $\mathrm{Al}$ cations could be tetrahedrally coordinated in sheet-like arrays [33]. This structural model is however not in accord with the atomic arrangements proposed by Kenway for the $\Sigma=11$ grain boundary.

Other useful sources of standard "fingerprints" are to be found in Al containing minerals. Brydson and co-workers have investigated the characteristic near-edge structures for a series of different $\mathrm{Al}$ coordinations $[8,34]$. Whilst none of the reported edge-shapes fit the grain-boundary "difference" data as well as that of the amorphous alumina, 4 (and possibly 5 fold) coordinated $\mathrm{Al}$-ions do exhibit the same characteristic features, including a weak leading shoulder at $77 \mathrm{eV}$ (in some cases this is resolved into a peak) followed by a stronger second peak in the $79 \mathrm{eV}$ region. They would correspond to features A and B in the standard "difference" spectra illustrated in figure 2. The calculated near-edge structure for a tetrahedrally bound $\mathrm{Al}$ cluster, reproduced from reference [8], is illustrated in figure 5 .

A second approach to verify the existence of the Kenway-structure, composed of a mixture of different "coordinations", would involve comparing the ELNES to detailed electronic structure calculations. To date, there have been no suitable grain-boundary calculations. The best that is currently available are the recent XANES computations for 4, 5 and 6 fold coordinated clusters, 
which are formally equivalent to real space K.K.R. band-structure calculations [8, 34]. According to these calculations, the first weak shoulder (feature-A) at about $76 \mathrm{eV}$ is due to a s-like final state and the second more intense absorption (B) at $78 \mathrm{eV}$ is due to "dipole forbidden" p-to-p transitions; the dipole selection rule being broken for systems lacking a center of inversion. A alternative view, based on the MO calculations for tetrahedral clusters [35], assigns the intensity of peak A at $76 \mathrm{eV}$ to "forbidden" transitions to $6 \mathrm{t}_{2}$ final states (composed predominantly of $\mathrm{Al}$ $3 p$ orbitals) and peak-B at $79 \mathrm{eV}$ to transitions to $6 a_{1}$ final states (predominantly of $s$ character) $[10,20,22]$. This assignment was used for the edge-structure of $\gamma$ and amorphous $\mathrm{Al}_{2} \mathrm{O}_{3}$ phases. By comparison to these theories, the oscillator strength observed at $76 \mathrm{eV}$ (region-1 in fig. 4) in the grain-boundary "difference" spectrum would be due to either unoccupied p-states (following Ref. [35]) or s-states (following Refs. [8] and [34]).

Following Bianconi $[10,21]$, the broad absorption peak lying in the $99-100 \mathrm{eV}$ energy-loss regime (peak C in Fig. 2) is an "inner well-resonance", produced by back-scattering of the excited electron having d-symmetry by the first near neighbor anionic oxygen shell [21]. A simple relationship existing between the resonance-energy, $E_{\mathrm{C}}$, relative to the Fermi-level, $E_{\mathrm{F}}$, and the anionic shell radius, $r_{\mathrm{c}}$, has been derived by Natoli [36] and is given by

$$
E_{\mathrm{C}}-E_{\mathrm{F}}=\frac{k}{r_{\mathrm{c}}^{2}}
$$

where $k$ is a system dependant constant. The assumption is made that both the Fermi-level, equal to $74.7 \mathrm{eV}$, and the value of $k$ remain constant in the bulk and at the boundary. At the near- $\Sigma=11$ grain boundary, the observed $0.9 \mathrm{eV}$ downward shift in the peak resonance energy suggests that the average $\mathrm{Al}-\mathrm{O}$ distance is increased relative to the $0.186 \mathrm{~nm}$ nearest neighbor distance of bulk sapphire by about $2 \%$ and the $5 \mathrm{eV}$ increase to the peak broadening corresponds to a $12 \%$ spread in the distribution of these lengths. For a Gaussean distribution of Al-O lengths at the boundary, the mean bond-length equals $0.189 \mathrm{~nm}$. These values compare favorably with the distribution of $\mathrm{Al}-\mathrm{O}$ bond-lengths calculated by Kenway. In his structure the mean $\mathrm{Al}-\mathrm{O}$ distance is $0.188 \mathrm{~nm}$ with standard deviation of $0.008 \mathrm{~nm}$.

A further comment on the Kenway model concerns the repeat distance projected along the [0111] direction (i.e. perpendicular to the viewing direction). This length is $1.335 \mathrm{~nm}$ and is similar to the experimental resolution which may account for the slight variability of the "difference" spectra. Within the boundary unit cell area of $0.482 \mathrm{~nm}$ by $1.335 \mathrm{~nm}$ there are 12 distinct $\mathrm{Al}$ cation sites.

Another possibility is the presence of a thin amorphous film at the near $\Sigma=11$ grain boundary film, a notion that would appear to be supported by the similarity of the "difference" spectra and the amorphous phase. This alternative, however, has been ruled out by the HREM studies [6]. No amorphous phase was ever observed at the atomically flat interface. In addition, the reproducibility of the "difference" spectra even for the shortest acquisition times suggests that the spectra are intrinsic features of the grain-boundary and not the result of amorphous phase formation due to specimen damage during the SREELS experiment.

An alternative description of the boundary is one in which the boundary plane comprises of either pure tetrahedral or mixed tetrahedral and octahedral units in a "spinel-like" structure. This would be analogous to the surface structure model based on a monolayer of spinel, proposed in reference [37] to account for the reconstruction of the basal $\{0001\}$ surface of $\alpha-\mathrm{Al}_{2} \mathrm{O}_{3}$, observed by HREM. Alternatively, the stucture might resemble the tetrahedral sheet model, as proposed in reference [33] for amorphous $\mathrm{Al}_{2} \mathrm{O}_{3}$. As mentioned previously, XANES calculations for tetrahedrally bound Al ions resemble the grain-boundary sensitive "difference" spectrum (Fig. 5), adding support to this notion. However, it has not been possible to construct a simple spinel-like struc- 
ture for the near $\Sigma=11$ boundary which would be both low in energy and have the required projected potential to match the HREM images as already achieved for Kenway's model [6].

Using the bimodal model constructed from tetrahedral and octahedral clusters, an average coordination number of aluminium ions at the boundary can be estimated directly from the mean bond-length. From figure 7 of reference [33], a bond-length of $0.189 \mathrm{~nm}$ indicates $30 \%$ tetrahedral coordination and $70 \%$ octahedral and an average coordination number equal to 5.4.

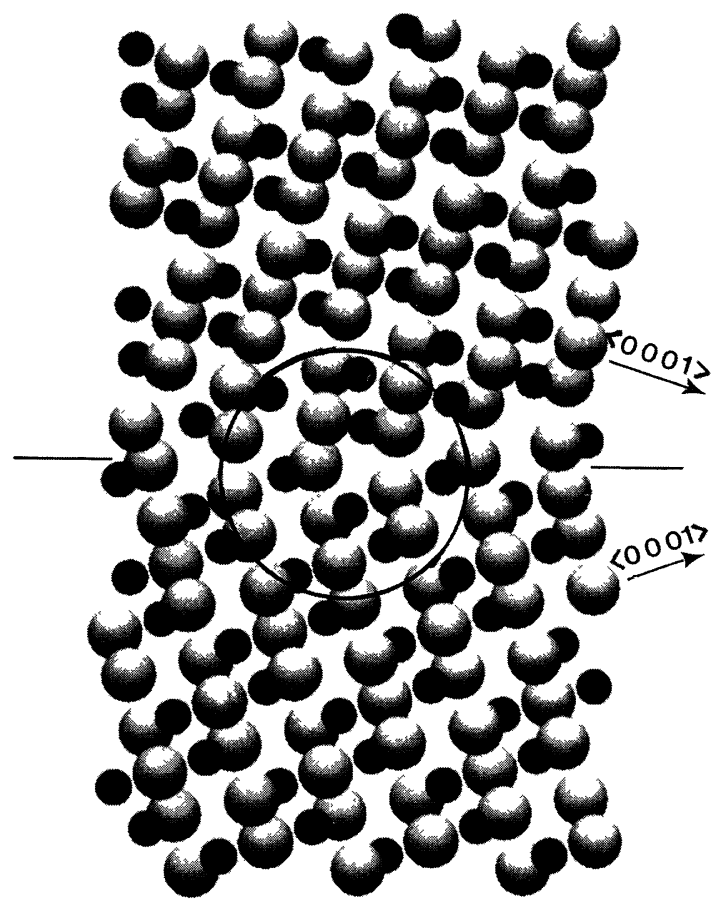

Fig. 7. - A projection of the relaxed Kenway structure of the near $\Sigma=1135.2^{\circ}[\overline{2} 110]$ tilt boundary. The boundary plane lies along the [0111] in this projection. The $\mathrm{Al}$ ions (dark circles) at the boundary are less densely surrounded by $\mathrm{O}$ anions.

The segregation of point defects, such as $\mathrm{O}$ vacancies or impurities, in the interface region as part of a space charge layer would be expected to influence the ELNES. Band-structure calculations predict the existence of a O-vacancy defect band extending below the conduction band minimum [28]. These would be detected as a pre-threshold shoulder on the $\mathrm{Al}_{2,3}$ edge. However, the formation energy for point defects of any kind in "pure" $\alpha-\mathrm{Al}_{2} \mathrm{O}_{3}$ is considered too high to lead to sufficient (i.e. a monolayer) intrinsic defects to have significant influence on the ELNES. Charged impurities reduce the defect formation energy and thus modify segregation in the space charge layer. It is known that some impurities such as $\mathrm{Si}$ and $\mathrm{Ca}$ strongly segregate to grain boundaries. Whilst small amounts of segregated impurity could influence the details of bonding at the boundary there is little evidence to suggest this is the case in an ultra-pure material with less than 59ppm total impurity content. Despite a careful search using EDX, EELS or HREM, there is no evidence to suggest impurities segregated to this $\Sigma=11$ boundary. 
The formation of an $\mathrm{Al}^{\delta+}$ sub-oxide phase was proposed to explain the appearance of a prethreshold shoulder on the $\mathrm{Al} \mathrm{L}_{2,3}$ edge of $\gamma-\mathrm{Al}_{2} \mathrm{O}_{3}$ in thermal oxidation studies of aluminium [38]. A chemical shift was attributed to a reduction in the $2 p$ core-level binding energy and the band-gap. A similar effect was also observed in silicon-rich-oxide structures [39]. There is no conclusive information currently available concerning the stoichiometry of the $\alpha-\mathrm{Al}_{2} \mathrm{O}_{3}$ close to this near CSL $\Sigma=11$ boundary. Surface techniques such as photoemission are not able to probe electronic structure of buried boundaries, thus it is not possible to determine the magnitude of the chemical shift, the position of the valence band edge, the band-gap, or the core-hole exciton binding energy.

\subsection{QUANTIFICATION OF THE INTERFACE COMPONENT.}

4.3.1 Detection sensitivity. - The standard treatment of the optimum detection sensitivity of a small signal superimposed upon a large background requires that the measured signal-to-noise ratio, $S N R_{0}$, be greater than a pre-selected level for positive identification [16]. A $99 \%$ confidence level of signal detection is achieved when $S N R_{0}$ exceeds 3 . Error in the background fitting increases the uncertainty in the resultant signal intensity, which adds to the error due to limited counting statistics. As Egerton described, this may be accounted for through a multiplicative factor, $h$, to the statistical noise. The $S N R_{0}$ and detection limit is conveniently expressed by the relation

$$
S N R_{0}=\frac{l_{x}}{\sqrt{h \cdot l_{\mathrm{b}}}} \cdot \sqrt{D Q E} \geq 3
$$

where $l_{x}$ is the interface sensitive signal, $l_{\mathrm{b}}$ is the background signal, and $D Q E$ is the detective quantum efficiency for the spectrometer system. The $D Q E$ describes the amplification of noise by the detection system, which at high count rates (i.e. just below detector saturation) is limited by the channel-to-channel gain variations.

For $l_{\mathrm{b}}$ equal to $2 \times 10^{5}$ accumulated electrons per channel, the $D Q E$ for the Gatan system is 0.05 [15]. If the background is known perfectly the standard error is limited only by the counting statistics of two spectra and the $h$-factor approaches a lower limit of 2 . Without a suitable chemical-standard spectrum it would be an almost impossible task to fit the rapidly varying background, resulting in an extremely high value of $h$ and a commensurately low detection sensitivity. Substituting these values of $l_{\mathrm{b}}, h$, and $D Q E$ into equation (2) indicates that $l_{x}$ should exceed about 8485 incident electrons per channel for positive signal identification (or about $4 \%$ of the total signal). Recording multiple spectra with such count rates introduces minimal additional noise, and the detection limit is further reduced by a factor equal to the square root of the number of readouts (i.e. $n$ readouts improves sensitivity by $\sqrt{n}$ times).

In the thin-film limit, $l_{x}$ is directly related to the number of atoms illuminated by the beam and is given by the expression

$$
l_{x}=\left(\frac{\mathrm{d} \sigma}{\mathrm{d} E}\right)_{x} \cdot \mathrm{d} E \cdot N_{x} \cdot D_{0}
$$

where $(\mathrm{d} \sigma / \mathrm{d} E)_{x}$ is partial ionization cross-section for atomic species $x, \mathrm{~d} E$ is the energy interval per channel, and $N_{x}$ is the number of atoms per unit area exposed to the electron beam, and $D_{0}$ is the incident number of primary electrons. For a partial cross-section equal to about $7 \times 10^{-26} \mathrm{~m}^{2} / \mathrm{eV} /$ atom (calculated for the $\mathrm{Al}-\mathrm{L}_{2,3}$ edge close to the threshold energy using the SIGMAL routine [16]), an incident dose of $1.5 \times 10^{10}$ electrons (equivalent to a 5 second exposure with a $0.5 \mathrm{nA}$ beam current) and $l_{x}$ equal to 8485 , the minimum value of $N_{x}$ with a $0.1 \mathrm{eV} / \mathrm{channel}$ dispersion given by equation (3) is 81 atoms per square nanometer. A $20 \mathrm{~nm}$ thick, sapphire foil 
would produce the background signal of $2 \times 10^{5}$ electrons assumed in this estimate, if the signalto-background jump-ratio at the $\mathrm{Al}-\mathrm{L}_{2,3}$ edge equals 2 .

The detection limit of 81 atoms $/ \mathrm{nm}^{2}$ is given with only one channel is used to identify the element. By evaluating the integral over 200 channels, the minimum sensitivity is improved by a factor of $\sqrt{200}$ to below 5.7 atoms $/ \mathrm{nm}^{2}$. Thus, near single atom sensitivity is achieved with a 5 second exposure time. To measure the near-edge structure (which typically has a dynamic range greater than 10) in the same energy-loss region requires about 10 times the "minimum-signal" intensity per channel and consequently 10 times as many atoms. Thus, from the spectrum generated by 6 multiple readouts about $10 \times 81 / \sqrt{6}=330$ atoms $/ \mathrm{nm}^{2}$ would be required for analysis of bonding using ELNES, which amounts to a concentration of about 14 at. \% in sapphire.

Such estimates depend critically upon the assumption that the signal can be accurately extracted from the background, i.e. setting $h=2$. A systematic error in the background fit will inevitably lead to an increase in $h$ and thus an increase in the minimum detectable number. They also are dependant upon the value of ionization cross-section which could be in error by an order of magnitude for energies close to absorption threshold. In the ELNES region, partial cross-sections are particularly sensitive to the final density-of-states and the transition matrix elements.

4.3.2 Quantitative analysis. - For oxides having similar atomic structure and composition the partial cross-section (integrated over a $30 \mathrm{eV}$ wide window) is expected to remain approximately constant. The total number of atoms illuminated by the incident beam is estimated from the foil thickness and the approximate diameter of the beam. The possible contributions to the effective probe size include the instrumental probe size, the inelastic interaction parameter and the beam spreading due to elastic scattering. The instrumental contribution to the probe diameter (containing $90 \%$ of the current) is about $0.9 \mathrm{~nm}$ [40]. The finite entrance aperture of the spectrometer (semi-angle equal to $8 \mathrm{mrad}$ ) excludes the contribution of electrons elastically scattered outside the collection angle, and so for a $20 \mathrm{~nm}$ thick foil, the cut-off diameter is equal to $20 \times 16 \times 10^{-3}=0.16 \mathrm{~nm}$. Recent investigations indicate that the inelastic impact parameter based on the average momentum transfer is not a significant factor [41]. The effective beam diameter for a fixed probe is about $0.96 \mathrm{~nm}$. In this case, the incident beam samples a total volume of about $14.3 \mathrm{~nm}^{3}$ and an area of grain boundary equal to about $19.2 \mathrm{~nm}^{2}$. This volume and area are occupied by about 500 atoms of $\mathrm{Al}$ in the corundum structure and 180 atoms in Kenway's grain-boundary structure model respectively.

The approximate number of $\mathrm{Al}$ atoms giving rise to the measured residual component may be estimated directly from the "difference"-to-bulk intensity ratio (values given in Tab. I). Because of the extreme assumptions, one would not expect an accuracy of better than $50 \%$ in this estimate. If $500 \mathrm{Al}$ atoms in the corundum structure were sampled by the beam, the average intensity ratio of 0.38 indicates that 190 cations are affected significantly by boundary. This amounts to about a monolayer of $\mathrm{Al}$ cations at the grain-boundary, supporting the notion that every $\mathrm{Al}$ atom on the near $\Sigma=11$ boundary plane occupies a site whose symmetry and near neighbor coordination is significantly different from that of the corundum structure. The variability in the experimental average intensity ratio probably indicates variability in the probe size which is sensitive to focus and mode of spectrum acquisition. A monolayer of ' $\mathrm{Al}$ ions agrees with the Kenway structure and is consistent with the estimate for the minimum number of atoms required to carry out ELNES analysis.

\section{Conclusions.}

A bi-crystal possessing a near CSL $\Sigma=11,35.2^{\circ}[\overline{2} 110]$ tilt boundary on the (0111) plane was examined by spatially resolved energy loss spectroscopy. The same boundary and sample had 
previously been examined in the high resolution microscope to investigate atomic structure. Here, focus was placed on the effect of the boundary on the near-edge structure on the $\mathrm{Al}-\mathrm{L}_{2,3}$ edge. Using the chemical standard "difference" method a contribution of the modified atomic structure was extracted.

The ELNES of the "difference" component is remarkably similar to that of amorphous alumina, indicating both materials ought to posses a similar short range order around the cation sites. There are various structural configurations which are possible. The one considered to be most likely is the model due to Kenway in which there are 12 distinct cation sites at the grainboundary, having a reduced point group symmetry and a modified coordination shell. Both the near-edge structure and the semi-quantitative analysis support the notion that a full monolayer of $\mathrm{Al}$ atoms at the boundary are affected. The detailed electronic band structure or chemical bonding at the grain boundary has not yet been calculated, however by comparison to spectra from known structures and XANES calculations this structure will probably not be extremely different from that obtained for four-fold or five-fold coordinated clusters, $\gamma-\mathrm{Al}_{2} \mathrm{O}_{3}$ or amorphous material.

\section{Acknowledgements.}

The author wishes to thank Dr. Kenway for providing critical details of his calculations prior to publication, Mr T. Höche and Dr. A. Kleebe for the provision of their TEM prepared sample, and R. Brydson for permission to reproduce the results of his XANES calculations. Comments and valuable discussions were provided by Dr. H. Müllejans and support from Professor Rühle is gratefully acknowledged. Funding for the purchase of the PEELS spectrometer was provided by a grant from the Volkswagen Stiftung.

\section{References}

[1] BATSON P.E., KAVANAGH K.L., WoNG C.Y. and Woodall J.M., Ultramicroscopy 22 (1987) 89.

[2] CollieX C., in "Transmission Electron Energy Loss Spectrometry in Materials Science" Disko, Ahn and Fultz Eds., The Minerals, Metals and Materials Society (1992).

[3] KRIVANEK O.L., MORY C., TENCÉ M. and CollieX C., Microsc. Microanaly. Microstruct. 2 (1991) 257.

[4] BRULEY J. and BATSON P., Phys. Rev. B 40 (1989) 9888.

[5] KENWAY P.R., private communication (1992).

[6] HÖCHE T.H., KENWAY P.R., KLEEBE H.J. and RÜHLE M., Proceedings of MRS Fall Meeting (1992).

[7] HOFER F., Habilitationschrift, Technischen Universität Graz (1988).

[8] BRydson R., Williams B.G., Engel W., Lindner T., Muhler M., SChlögl R., Zeitler E. and THOMAS J.M., J. Chem. Soc. Faraday Trans. 84 (1988) 631.

[9] BRYDSON R., EMSA Bull. 21(2) (1991) 57-67.

[10] BianConi A., Surface Sci. 89 (1979) 41-50.

[11] LARTIGUe S. and PRIESTER L., J. Phys. Colloq. 46 (1985) C4-101.

[12] FoRTUNEE R.P., M.S. Thesis, Case Western Reserve, Cleveland (1981).

[13] MORRIS P.A., Ph.D., MIT (1986).

[14] BONEVICH J.E. and MARKS L.D., Ultramicroscopy 35 (1991) 161.

[15] KRIVANEK O.L., AHN C.C. and KEENEY R.B., Ultramicroscopy 22 (1987) 103.

[16] EgERTON R.F., "Electron energy-loss spectroscopy in the electron microscope" Plenum Press, New York (1986).

[17] Shuman H. and SomLYo A.P., Ultramicroscopy 21 (1987) 23.

[18] BRULey J., Phil. Mag. Lett. 66(1) (1992) 47.

[19] Colliex C., Manoubi T., Gasgnier M. and Brown L.M., Scann. Electr. Micr. 2 (1985) 489.

[20] Brytov I.A. and RomasChEnKo Y.N., Sov. Phys. Solid State 20(3) (1978) 384. 
[21] Bianconi A., in "EXAFS and Near Edge Structure", A. Bianconi, L. Incoccia and S. Stipcich Eds., Springer Ser. Chem. Phys. Vol. 27, (Springer-Verlag (1983) p. 118.

[22] Balzorotti A., ANtonangeli F., Girlanda R. and MARTino, Phys. Rev. B. 29 (10) (1984) 5903.

[23] El-MASHRI S.M. and FORTY A.J., in "Electron Microscopy and Analysis 1981" (Institute of Physics, 1982) p.395.

[24] Tossell J.A., J. Phys. Chem. Sol. 36 (1975) 1273-80.

[25] Batra I.P., J. Phys. C. (Solid State Phys.) 15 (1982) 5399.

[26] OHUCHI F.S. and KoHYAMA M., J. Am. Cer. Soc. 74(6) (1991) 1163.

[27] Shangda X., ChandXIN G., Libin L. and Ellis D.E., Phys. Rev. B 35(14) (1987) 7671.

[28] CIRACI S. and BATRA I.P., Phys. Rev. B 28(2) (1983) 982.

[29] XU Y.-N. and CHING W.Y., Phys. Rev. B 43(5) (1991) 4461.

[30] Guo J., Ellis D.E. and LAM D.J., Phys. Rev. B 45(23) (1992) 13647-56.

[31] Balzorotti A., ANTONANGeli F., Girlanda R. and Martino G., Solid State Commun. 44(2) (1982) 275.

[32] OLIVIER J. and POIRIER R., Surface Sci. 105 (1981) 347.

[33] El-MASHri S.M., JONES R.G. and FoRTY A.J., Phil. Mag. A 48(5) (1983) 665.

[34] HANSEN P.L., MCCOMB D.W., BRYDSON R. and RICHARDSON I., private communication (1992).

[35] Tossell J.A., J. Amer. Ceram. Soc. 97(17) (1975) 4840.

[36] NATOLi C.R., in "EXAFS and Near Edge Structure”, A. Bianconi, L. Incoccia and S. Stipcich Eds., Springer Ser. Chem. Phys. Vol. 27 Springer-Verlag (1983) p.43.

[37] BURSILl L.A. and LiN P.J., Phil. Mag. 60(3) (1989) 307.

[38] BianCONi A., BACHRACH R.Z., HAGSTROM S.B.M. and FlodSTRÖM S.A., Phys. Rev. B 19(6) (1979) 2837.

[39] Dori L., Bruley J., DiMaria D.J., Batson P.E., Tornello J. and Arienzo M., J. Appl. Phys. 69(4) (1991) 2137.

[40] MORY C., Ph.D. Thesis, Université de Paris-Sud, Orsay (1985).

[41] MORY C., Kohl H., TENCÉ M. and COLLIEX C., Ultramicroscopy 37 (1991) 191. 